\title{
Predictors of Successful Outcomes Following Revision Total Knee Arthroplasty for Flexion Instability
}

Payton K Arnold ${ }^{1}$, Joseph A Madden', Mary Ziemba-Davis ${ }^{2}$, R. Michael Meneghini MD²,3

${ }^{1}$ Indiana University School of Medicine

2 Indiana University Health Physicians, Orthopaedics

${ }^{3}$ Indiana University School of Medicine, Department of Orthopaedic Surgery

Background and Hypothesis: Flexion instability is a poorly understood cause of total knee arthroplasty (TKA) failure, often referred to as a "wastebasket" diagnosis for unexplained persistent pain. It remains a challenging diagnosis, with unpredictable outcomes following revision surgery. This study identified predictors of successful patient reported outcome measures (PROMS) following revision for flexion instability.

Methods: 121 consecutive revision TKAs for flexion instability from 2011-2018 at a single center were retrospectively reviewed. PROMS were prospectively obtained preoperatively and at minimum one-year follow-up. 33 potential predictors of PROMS encompassed: presenting symptoms, demographics/medical covariates, intraoperative observations, and pre/postoperative radiographic measurements. Variables related to outcomes with $p \leq 0.20$ in univariate analysis were entered in regression models. Nonsignificant predictors were pruned until only predictors with $p<0.05$ and the best model statistics were achieved.

Results: The sample was $63 \%$ female with mean age of $65 \pm 11$ and BMI of $34 \pm 7$ $\mathrm{kg} / \mathrm{m}^{2}$. Pre- to postoperative changes in three radiographic measurements vital to the stability and function of TKA were related to function $(p<0.001)$, pain $(p=0.019)$, and satisfaction $(p=0.030)$ after surgery. Pain with walking $(p \leq 0.059)$ and stair climbing $(p \leq 0.048)$ were almost exclusively related to demographic/medical covariates. Only one of the presenting symptoms commonly associated with flexion instability influenced early revision outcomes $(p=0.021)$.

Conclusion and Potential Impact: Instability is one of the most common reasons for TKA failure leading to costly revision surgery. To our knowledge, this is the largest flexion instability cohort studied to date, including substantially more predictors in an attempt to elucidate factors indicative of successful treatment outcomes. With the exception of radiographic parameters reflecting surgical decisions, findings suggest that many factors related to outcomes may be beyond the control of arthroplasty surgeons. 